\title{
Tracking of the Left Ventricle in Contrast Enhanced Echocardiography by Anisotropic Filtering and Active Contours Algorithm
}

\author{
MA Morales, V Positano, O Rodriguez, M Passera, M Lombardi, D Rovai \\ CNR Clinical Physiology Institute, Pisa, Italy
}

\begin{abstract}
Contrast agents together with second harmonic imaging improve endocardial border detection in echo images. Aim of the study was to assess whether non linear anisotropic filtering after segmentation of gradient vector flow $(G V F)$ based active contour algorithm can be applied on contrast echo images for automatic detection of left ventricular $(L V)$ contours. $L V$ volumes were measured in 20 subjects by cine Magnetic Resonance (MR) and transthoracic second harmonic echo after intravenous administration of the contrast agent Levovist. Contrast echo images were analyzed both manually and automatically; $M R$ images were automatically analyzed. LV volumes were calculated according to Simpson's rule. A good correlation with MR volumes was found for manual LV volumes (r.93 and .92 for end diastolic and end systolic volumes, respectively, $p$ $<.05)$. A still good correlation was found for the automatically assessed volumes in both phases ( $r=.90$ and .91, $p$ <05). Thus automatic endocardial border delineation by anisotropic filtering and GVF based active contour algorithm is feasible on contrast echo images. This method may represent an approach for automatic endocardial border analysis in ultrasonic imaging.
\end{abstract}

\section{Introduction}

The intravenous injection of ultrasound contrast agents able to cross the lungs determines an enhancement of the echocardiographic signal reflected from left ventricular (LV) cavity improving the delineation of endocardial borders $[1,2]$. This approach allows to investigate LV volumes and function, as well as regional wall motion abnormalities, also in patients with suboptimal image quality [3-5]. So far in the clinical setting, despite several semiautomatic methods have become available, this anatomic and functional information has mostly been obtained by tracing endocardial borders manually. Active contour models (snakes) are extensively used in medical image analysis [6] to process MR, CT and ultrasound images $[7,8]$. Aim of this study was to evaluate a new approach for automated endocardial border detection in contrast-enhanced echocardiographic images. This method is based on an implementation of the original snake algorithm. LV volumes obtained by automated cine magnetic resonance were the golden standard for the study.

\section{Materials and methods}

\subsection{Patient population}

Twenty patients (18 men, 2 women), undergoing cardiac magnetic resonance imaging for clinical purposes were enrolled in the study. The mean age was $57 \pm 12$ years. LV function was normal in 7 patients and abnormal in 13, without distortion of LV shape.

\subsection{Cine magnetic resonance imaging}

All exams were performed by a GE CVi 1.5 TESLA equipped with phased array chest coil. MRI images for ventricular volumes calculation were acquired using FIESTA sequence that allows to obtain over 30 phases of cardiac cycle in a single slice within 10-15 seconds. Parallel slices (up to 15) of the ventricle in short axis were acquired to cover the entire volume of the ventricles (slice thickness $8 \mathrm{~mm}$, no interslice gap). A semiautomatic program (MASS Analyser) was used to identify the endocardial and the subepicardial borders and to compute the $\mathrm{LV}$ volumes.

\subsection{Contrast echocardiography}

Echocardiography was performed with commercially available equipment (General Electric, Vingmed System 5, Norway). After a complete echocardiographic examination in fundamental imaging, the scanner was set in harmonic imaging, with the transducer transmitting at 1.6 $\mathrm{MHz}$ and receiving at 3.2 MHz. A galactose-based contrast agent (Levovist, Schering AG) was intravenously injected as a slow bolus (4 grams at a concentration of $400 \mathrm{mg} / \mathrm{ml}$ ). All echo images were recorded in digital format and stored on optical disk for subsequent analysis. 


\subsection{LV contour analysis and volume calculations}

\section{Manual contour detection.}

Digitized images were reviewed by two experienced cardiologists who manually drewthe LV contours and calculated LV volumes according to the modified Simpson's rule.

\section{Automatic contour detection}

The original snake algorithm was improved using the adaptive anisotropic filter [9] that:

a) removes noise in regions of homogeneous physical properties (i.e. contrast medium), thus improving signal to noise ratio and contrast;

b) enhances morphological definition by sharpening organ discontinuities.

The filtering method is described by the following equation

$$
\frac{\partial}{\partial t} I(\mathbf{x}, t)=\operatorname{div}[c(\mathbf{x}, t) \cdot \nabla I(\mathbf{x}, t)]
$$

The diffusion strength is controlled by the conductance function $\mathrm{c}(\mathbf{x}, \mathrm{t})$. The vector $\mathbf{x}$ represents the spatial coordinate, while the variable $t$ in our discrete implementation corresponds to iteration step $n$. The function $I(x, t)$ is the image intensity. Choosing a decreasing conductance function of the image gradient, the diffusion is reduced or even blocked when close to an image discontinuity. The choice of the conductance function is critical for effectiveness of the algorithm and depends on nature of the images. For contrast enhanced cardiac US images we use [10]:

$$
c(\mathbf{x}, t)=\frac{1}{2}[\tanh (\gamma(k-\|\nabla I(\mathbf{x}, t)\|))+1] .
$$

The parameters $\gamma$ control the steepness of the min-max transition region, whereas $k$ controls the extent of the diffusion region in terms of gradient gray-level. $\gamma$ can be fixed to 0.2 for 256 gray-level images. In medical images processing, the $\gamma$ value should be incremented proportionally to the range of the image values.

Starting from pre-filtered images, a deformable model [11] was developed as a curve that moves through the spatial domain of an image to minimize the following energy functional:

$$
E=\int_{0}^{1} \frac{1}{2}\left[\alpha\left|\mathbf{x}^{\prime}(s)\right|^{2}+\beta\left|\mathbf{x}^{\prime \prime}(s)\right|^{2}+E_{\text {ext }}(\mathbf{x}(s))\right] d s
$$

where: $\quad \mathbf{x}(s)=[x(s), y(s)]$ and $\mathrm{s} \varepsilon[0,1], \quad \alpha$ and $\beta$ control the mechanical properties of the snake, i.e. tension and rigidity respectively, $\mathbf{x}^{\prime}$ and $\mathbf{x}^{\prime}$ ' denote the first and the second derivatives of $\mathbf{x}(\mathrm{s})$ with respect to $\mathrm{s}$, and $E_{\text {ext }}(\mathbf{x})$ is the potential associated to the external forces. $E_{\text {ext }}(\mathbf{x})$ is derived from the image gradient so that it takes on its smaller values at the edge points. The external force can be defined to be a vector field $\mathbf{v}(\mathbf{x})$ that minimizes the following function:

$$
\varepsilon=\iint \mu|\nabla \mathbf{v}|^{2}+|\nabla I|^{2}|\mathbf{v}-\nabla I|^{2} d \mathbf{x}
$$

The external field $\mathbf{v}(\mathbf{x})$ resulting from this calculation of variations is used in E expression as potential force $\nabla \mathrm{E}_{\mathrm{ext}}(\mathbf{x})$, yielding:

$$
\mathbf{x}_{t}(s, t)=\alpha \mathbf{x}^{\prime \prime}(s)-\beta \mathbf{x}^{\prime \prime \prime}{ }^{\prime}(s)+\mathbf{v}
$$

where $\mathbf{X}^{\prime \prime}$ and $\mathbf{X}^{\prime \prime \prime \prime}$ are respectively the second and the fourth derivative of $\mathbf{x}(\mathrm{s})$ with respect to $\mathrm{s}$. The GVF snake is represented by the parametric deformable surface solving the previous equation [12].

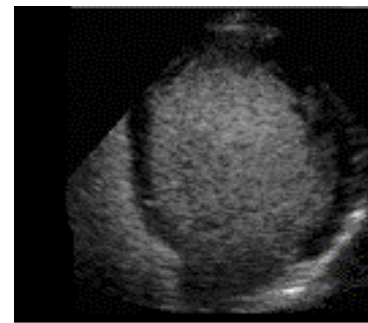

(a)

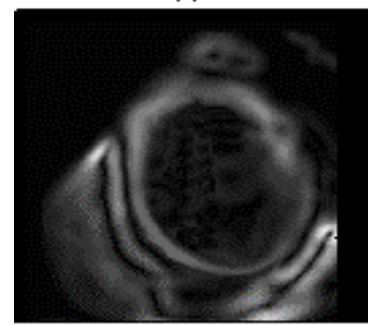

(c)

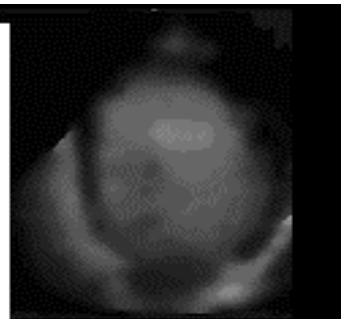

(b)

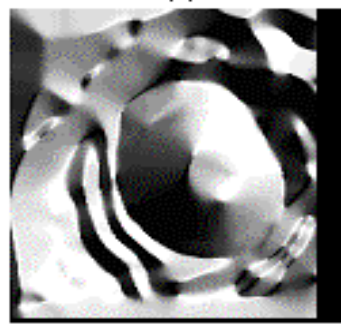

(d)
Figure 1. Algorithm phases: (a) starting image, (b) filtered image; (c) Gradient image; (d) GVF image.

Step-by-step analysis

1. Application of the $2 \mathrm{D}$ nonlinear anisotropic filtering to echocardiographic images (Fig 1.b).

2. Initialization of GVF snake procedure by manual tracing of a very rough close curve inside the left ventricular cavity in the $2 \mathrm{D}$ image.

3. Fitting of left ventricular border with a GVF snake made by the evaluation of the GVF field (Fig 1.d) 
starting from the filtered image edge map (Fig 1.c). From the initial curve, the snake evolves fitting the LV cavity.

4. Manual tracing of the LV axis. LV volume is calculated by the modified Simpson's rule.

In Figure 2, the first minimum of GVF field (a) is over-imposed on the original image. The minimum of the GVF field constitutes a good approximation of LV contour.

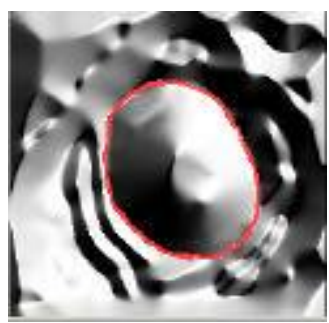

(a)

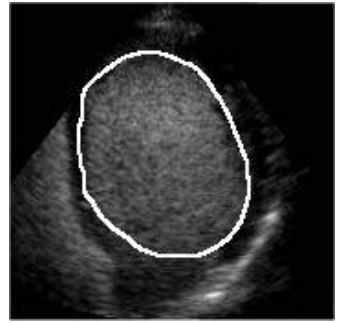

(b)
Figure 2.Coincidence between the first minimum of GVF field (a) and the LV contour (b).

\section{Results}

A good correlation was found between manually extracted LV end diastolic and end systolic volumes and cine MRI, being the correlation coefficient $r$ equal to 0.93 and 0.92 for EDV and ESV, respectively (Fig 3). A worse, but still good correlation was also reported when EDV (Fig. 4) were assessed by the automatic contour detection method. When the Bland Altman method was applied [13] the volumes calculated by MRI were slightly bigger than those obtained by contrast echo, both manually and automatically analyzed., as shown for EDV in Fig 5.

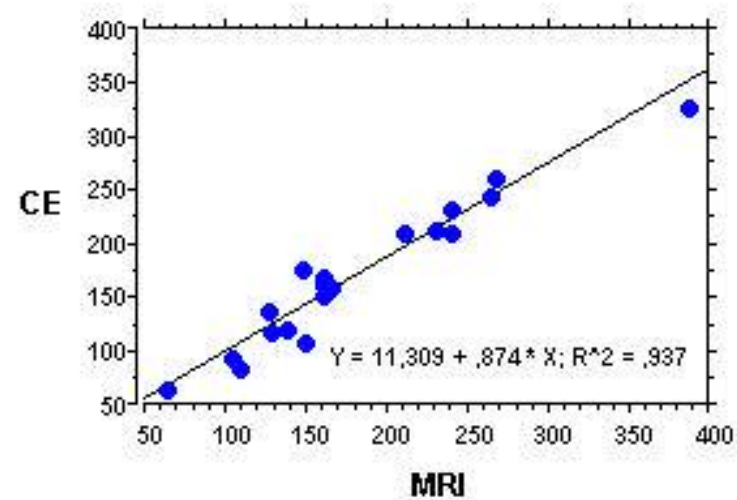

Figure 3. CE-MRI correlation for manual analysis of enddiastolic volumes.

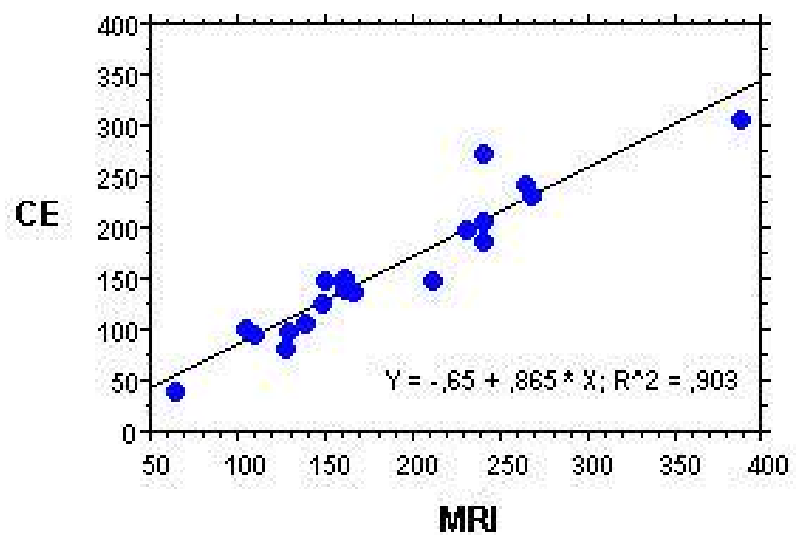

Figure 4. CE-MRI correlation for automatic analysis of enddiastolic volumes.
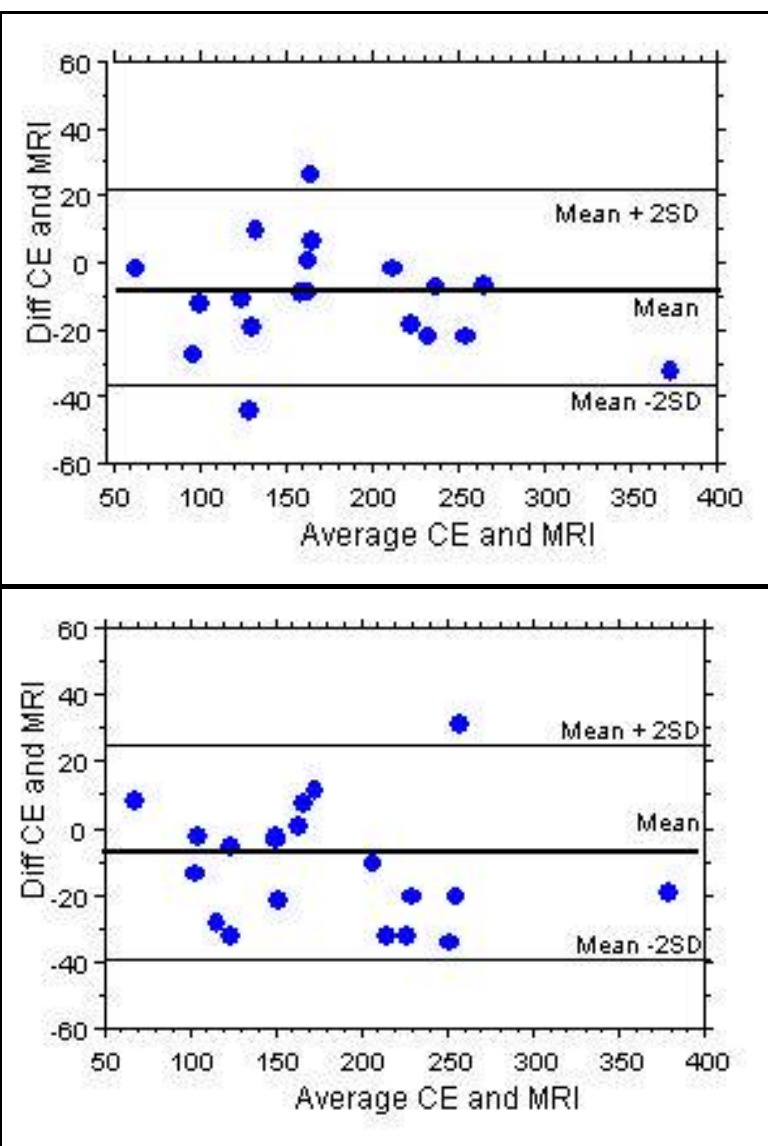

Figure 5. Bland Altman analysis for manual (upper) and automatic (lower) analysis of end-diastolic $L V$ volumes on CE images

\section{Conclusions}

The combination of intravenous contrast administration and second harmonic imaging was able to determine opacification of the left ventricle in all the studied 
patients. Although a better correlation with MR volumes was found for manually outlined ventricles, the automatic definition of the endocardial borders still gave a good relation. From these data it is evident that MR volumes are bigger than the echo ones, especially for subjects with abnormal LV function. A possible explanation is that contrast may reduce the visualization of the mitral annular plane as well as of the endocardium at lateral wall level leading to some underestimation of contrast enhanced LV volume (14).

The GVF field represented a good choice for external forces field in the snake algorithm: a maximum is present in the center of LV shape and the field slowly degrades until a minimum, in correspondence of the LV contours. Therefore, almost any snake traced in the LV cavity converged to the LV contour. The main advantage of this algorithm is to preserve the continuity of the detected contour, respecting the anatomical characteristics of the cardiac walls. The anisotropic filtering allows to overcome the problems related to the high level of corrupting noise, so that it is no longer mandatory to place the snake close to the left ventricular outline.

In conclusion, the combination of the anisotropic filtering of images and the active contour analysis seems effective in processing contrast enhanced US images, allowing automatic analysis and robust convergence to the LV contours in both systolic and diastolic phases.

\section{References}

[1] Lindner JR, Dent JM, Moos SP, Jayaweera AR, Kaul S. Enhancement of left ventricular cavity opacification after venous injection of Albunex. Am J Cardiol 1997; 79: 165762.

[2] Al-Mansour HA, Mulvagh SL, Pumper GM, Klarich KW, Foley DA. Usefulness of harmonic imaging for left ventricular opacification and endocardial border delineation by optison. Am J Cardiol 2000 Mar 15;85(6):795-9, A10.

[3] Cruise LJ, Cheirif J, Hanly de et al. Opacification and border delineation improvement in patients with suboptimal endocardial border definition in routine echocardiography. J Am Coll Cardiol 1993; 22: 1494-500.

[4] Van Camp G, Franken RR, Schoors D et al. Impact of second harmonic imaging on the determination of the global and regional left ventricular function by $2 \mathrm{D}$ echocardiography: a comparison with MIBI gated SPECT. Eur J Echocardiogr 2000 Jun;1(2):122-9.

[5] Senior R. Role of contrast echocardiography for the assessment of left ventricular function. Echocardiography 1999 Oct;16(7, Pt 2):747-752.

[6] Singh A, Goldgof D, Terzopoulos D. Deformable models in medical image analysis. IEEE press. 1998.

[7] Malassiotis S, Strintzis MG. Tracking the left ventricle in echocardiographic images by learning heart dynamics. IEEE Trans. on Medical Imaging, 1999;18(3):282-290.

[8] Mikic I, Krucinski S, Thomas JD. Segmentation an tracking in echocardiographic sequences: active contours guided by optical flow estimates. IEEE Trans. on Medical Imaging 1998;17(2): 274-284.

[9] Perona P, Malik J. Scale-Space and edge detection using anisotropic diffusion. IEEE Trans. on Pattern Analysis and Machine Intelligence 1990; 12(7):629-639.

[10] Monteil J, Beghadadi A. A new interpretation and improvement of the non linear anisotropic diffusion for image enhancement. IEEE Trans on pattern anlysis and machine intelligence 1990; 12(7): 940-946.

[11] Kass M, Witkin A, Terzopoulos D. Active contours models. Int J Computer Vision 1987; 1: 321-331

[12] Xu C and Prince JL. Snakes, shapes and gradient vector flow. IEEE Transactions on image processing. 1998;7(3) 359-69.

[13] Bland JM, Altman DG. Statistical methods for assessing agreement between two methods of clinical measurement Lancet i. 1986;307-10.

[14] Hundley WG, Kizilbash AM, Afridi I et al. Administration of an intravenous perfluorocarbon contrast agent improves echo determination of LV volumes and ejection fraction. Comparison with Cine MRI. J Am Coll Cardiol 1998; 32: 1426-32.

Address for correspondence:

Maria-Aurora Morales

IFC-CNR, Via Moruzzi, 1 - 56124 Pisa - ITALY

morales@ifc.cnr.it 